BBA $375^{\circ}$

\title{
AFTERGLOW OF CHOROPHYLL IN VIVO AND PHOTOSYNTHESIS
}

\author{
J. C. GOEDHEER
}

Biophysical Research Group, Institute of Physics, State University, Utrecht (The Netherlands)

(Received May 4 th, I962)

\section{SUMMARY}

Two pigment systems are involved in the afterglow of chlorophyll $a$-containing cells. Absorption in only one of these systems (promoting or "p" system) is effective in producing luminescence. If light is absorbed simultaneously by the other (quenching or " $q$ " system), a decrease in luminescence results. Absorption in the "p" system is assumed to result in oxidation of water and reduction of a cytochrome. The afterglow is regarded as a chemiluminescence of chlorophyll excited by the recombination of photolysis products of water.

Absorption in the " $q$ " system is assumed to oxidise cytochrome and reduce a pyridine nucleotide. In this way electrons are removed from the "p" system and recombination prevented. Each system provides about half the energy needed in complete photosynthesis.

Chloroplast luminescence is decreased by removal of electrons from the " $p$ " system by addition of Hill reaction reagents.

The "p" system includes mainly "short-wavelength chlorophyll $a$ " and the accessory pigments, the "q" system mainly "long-wavelength chlorophyll $a$ ".

Indications are presented that energy for both systems is provided by reversible photooxidation of chlorophyll $a$. The necessary separation between electrons leaving and electrons entering chlorophyll is assumed to result from a difference in molecular orbitals of ground and excited states, inferred from the differences between reversible photooxidation and photoreduction of chlorophyll in vitro.

The function of pigment systems in photosynthetic bacteria is discussed in re lation to those of green plants.

\section{INTRODUCTION}

STREHLER AND ARNOLD ${ }^{1}$ discovered that green plants and algae show an afterglow lasting several seconds after illumination. The intensity of the afterglow was about one millionth of that of absorbed light if this was below light saturation. The dependence of the luminescence on temperature, intensity of absorbed light, ultraviolet radiation, and inhibitors generally paralleled that of photosynthesis.

STREHLER AND ARNOLD suggested that the phenomenon was a chemiluminescence coupled with back reactions in the early steps of photosynthesis. They also suggested that probably one or more enzyme systems were involved in the luminescent system.

Abbreviations: PN, phosphopyridine nucleotide; PNH, reduced phosphopyridine nucleotide. 
ARNOLD $^{2}$ showed that light saturation of this luminescence occurs at a much lower intensity than that of photosynthesis.

ARnold AND DAvidson ${ }^{3}$ analysed the emission spectrum of the afterglow and found it to be similar to the emission spectrum of chlorophyll fluorescence. ARNOLD AND THOMPSON ${ }^{4}$ determined action spectra of luminescence of red and blue-green algae. These action spectra were found to be similar to those of photosynthesis.

ARTHUR AND STREHLER ${ }^{5}$, using a rotating-sector method, found the luminescence to be composed of a short-lived component of about o.oI sec and a component of a much longer life. The latter component was thought to be the luminescence measured by the flow system of STrehler And Arnold. Arthur And STrehler suggested that the component of shorter duration is determined by the concentration of the first oxidised and reduced products in photosynthesis, while the luminescence of longer duration might be due to a reducing intermediate formed later in the reaction chain. STREHLER AND LYNCH ${ }^{6}$ found a close correlation between the dependence on light intensity of chloroplast luminescence and a reversible change in absorption at $645 \mathrm{~m} \mu$.

ARNOLD AND SHERWOOD ${ }^{7}$ measured a thermoluminescence emitted by dried chloroplast powders. This luminescence showed the same characteristics as thermoluminescence emitted by crystalline inorganic systems. ToLlin AND CALvin ${ }^{8}$ measured luminescence emitted by a layer of chloroplasts at various temperatures between $23^{\circ}$ and $-\mathbf{I}_{4} \mathbf{O}^{c}$ after excitation with an intense flash of light. The low temperature curves were strictly exponential; at room temperature the afterglow showed a less rapid decay. These investigators also determined thermoluminescence curves. BRUGGER ANI) FRANCK ${ }^{9}$ investigated the influence of various cofactors and of $\mathrm{CO}_{2}$ on the time course of luminescence of algae and chloroplasts. Albrecht, Denison, Livingston ANI MANDEvilLE ${ }^{10}$ studied the quenching of the afterglow of Chlorella by light of $700 \mathrm{~m} \mu$. They noted a luminescence of chlorophyll $a$ in glassy cellulose butyrate lackers.

The authors of the latter four papers believe the afterglow to result from crystalline properties of chlorophyll layers in the chloroplasts. Chlorophyll $a$ and related pigments, however, are able to emit a chemiluminescence upon heating (chlorophyll and magnesiumphtalocyanine in tetralin $\left.{ }^{11}\right)$, which accompanies decomposition of peroxides $^{12}$ and is coupled with reversible oxidation ${ }^{13}$. The chemiluminescence mechanism thus deserves renewed study. Moreover, luminescence measurements can be used for studying the kinetics of photosynthetic reactions in an intensity range and with a time resolution not easily obtainable with other methods. The results of such a study are presented below.

\section{METHODS}

Most experiments were performed with a circulating-flow method analogous to the one used by STREHLER AND ARNOLD ${ }^{1}$. This method was chosen in order to approach as closely as possible conditions of continuous illumination.

If no cooling was applied, the afterglow was measured $0.2 \mathrm{sec}$ after illumination. When cooling was needed a thermostat was included in the assembly. The increased flow resistance due to the cooling spiral, resulted in a lengthening of the interval between illumination and detection to $0.5^{-I}$.o sec.

Luminescence was detected by a liquid air-cooled photomultiplier (Dumont 69II). The signal was fed into a direct current amplifier and recorded on a Brown recorder 
(Io $\mathrm{mV} \mathrm{I} \mathrm{sec} \mathrm{or} \mathrm{Io} \mathrm{mV} 0.25 \mathrm{sec}$ ). The illumination was carried out in a rectangular lucite cuvette by one or two incandescent lamps (I00 W, Io A) placed on opposite sides of the cuvette. Monochromatic light was obtained by using interference filters (half width $\mathrm{I} \circ \mathrm{m} \mu$ ) or a grating monochromator. The volume of the lucite cuvette was approximately four times the volume of the rest of the assembly. Thus, the average time the cell suspension was in darkness was about $20 \%$ of the total time of the experiment.

If cooling or heating was applied, care was taken that the temperature fluctuations in the circulating system did not exceed $3^{\circ}$.

Chloroplasts were obtained by mincing spinach leaves in a Brown multipress. They were suspended in o.or $M$ phosphate buffer ( $\mathrm{pH}$ 7.o) and used as a crude suspension or precipitated by centrifugation and resuspended in phosphate buffer (o.or $M, \mathrm{pH} 7.0$ ).

Oxygen evolution served as a measure of photosynthesis, using a polarographic method analogous to the one described by HAXO AND BLINKS ${ }^{15}$. In some experiments oxygen output was measured with a mass spectrometer: A measure of the Hill reaction was obtained optically by the reduction of the blue dye 2,6-dichlorophenolindophenol ${ }^{16}$.

The light intensity was measured with a thermopile. For these measurements infrared light was removed by a Calflex filter (cut-off at $700 \mathrm{~m} \mu$ ) combined with two Schott IR filters. A correction for the absorption of these filters in the visible part of the spectrum was applied. Intensities of white light, covering the spectral region from 400 to $700 \mathrm{~m} \mu$, are given in $\mathrm{ergs} / \mathrm{cm}^{2} \cdot \mathrm{sec}$.

A few experiments were performed with a rotating-sector phosphoroscope, the signal being fed into an oscilloscope. In order to avoid interference by scattered light, the incident beam was filtered. This resulted in a loss of a fraction of the light, particularly in the red.

\section{RESULTS}

\section{Luminescence as a function of intensity of white exciting light}

Data showing dependence of luminescence and photosynthesis on the intensity of incident light ("light curves") for Chlorella pyrenoidosa are presented in Fig. Ia. Both light curves are measured under the same conditions of temperature and $\mathrm{CO}_{2}$ content. Confirming ARNOLD's ${ }^{2}$ findings the figure shows that light saturation of Chlorella luminescence occurs at a much lower incident intensity than light saturation of photosynthesis. The luminescence maximum was much more pronounced with Synechococcus. No maximum was observed in photosynthesis.

Luminescence "light curves" of different cultures vary widely. Sometimes no maximum is present.

In Fig. Ib the "light curves" for luminescence and Hill reaction capacity of spinach chloroplasts are presented. In both cases saturation occurs at light intensities beyond $\mathrm{IO}^{4} \mathrm{ergs} / \mathrm{cm}^{2} \cdot \mathrm{sec}$, and there is no maximum. The luminescence "light curve" is found to be less steep then the Hill reaction curve.

The conditions under which the experiments with chloroplasts were performed were different from those employed with algal cells. With the latter the terminal electron acceptor $\mathrm{CO}_{2}$ was present in the same concentration in the luminescence 
and in the photosynthesis measurements; no electron acceptor necessary for the Hill reaction was added to the chloroplast suspensions in which luminescence "light curves" were measured. The following section may justify this procedure.

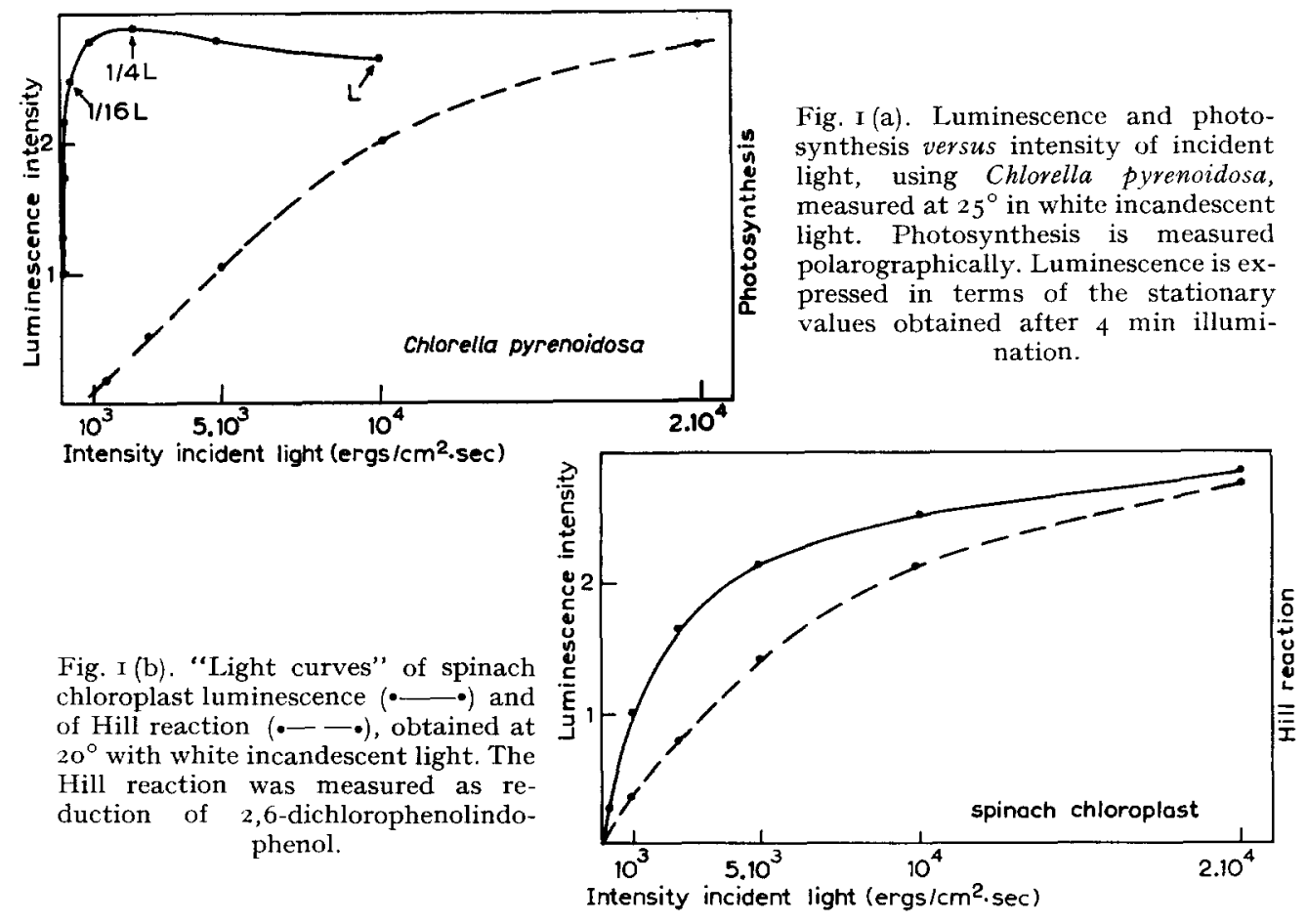

\section{Infuence of addition of oxidants and reductants on chloroplast luminescence}

If an oxidant, active in the Hill reaction, such as p-benzoquinone (in the following abbreviated to "quinone"), 2,6-dichlorophenolindophenol, or ferricyanide is added to a luminescent chloroplast suspension, the luminescence drops considerably and immediately. A 90\% drop was obtained after addition of $\mathrm{IO}^{-3} M$ quinone. The percentage drop in luminescence intensity was the same at incident intensities of $6 \cdot \mathrm{IO}^{3}$, $4 \cdot \mathrm{IO}^{2}$ and $50 \mathrm{ergs} / \mathrm{cm}^{2} \cdot \mathrm{sec}$. Hence the general shape of the light curve is most probably not affected by addition of oxidant.

If the added oxidant is reduced as a result of illumination, the luminescence increases. Upon addition of ascorbic acid, cysteine or sodium dithionite, luminescence increases immediately to practically its original value. Addition of these substances to chloroplast suspensions not containing an oxidant is without effect, provided washed chloroplasts are used and the solution is adequately buffered.

During the illumination of the chloroplast suspensions (without added oxidant) the capacity for luminescence drops slowly. The effects of addition of oxidants and reductants, however, do not change after partial inactivation by light.

\section{Infuence of temperature and storage on luminescence}

Influence of temperature was determined for Chlorella and Synechococcus. In these algal species the temperature curves for luminescence and photosynthesis were 
similar in shape, but showed relatively large quantitative variations. In general the temperature optimum of Chlorella luminescence was around $39^{\circ}$, and of Synechococcus around $29^{\circ}$. Heating to temperatures exceeding these values resulted in an irreversible decrease in luminescence capacity: e.g. $5 \mathrm{~min}$ storage at $65^{\circ}$ resulted in a decrease of the luminosity to less than I \% of its maximum value.

When establishing temperature curves the temperature had to be kept constant at each point for a few minutes. This was necessary because luminescence adjustment to temperature required $\mathrm{I}-2 \mathrm{~min}$.

In Fig. 2 temperature curves for luminescence and Hill reaction capacity of spinach chloroplasts are presented. The curves, measured with aliquots of the same suspension, show the same general shape. Also with chloroplasts luminosity had vanished after $5 \mathrm{~min}$ heating to $65^{\circ}$.

Storage in the dark at room temperatures $\left(18-20^{\circ}\right)$ for about $6 \mathrm{~h}$ did not result in a marked decrease either in luminescence or in Hill reaction activity. After $30 \mathrm{~h}$ storage both activities had decreased by $70-80 \%$. Suspensions of Aspidistra elatior leaves showed the same storage effects as those of spinach chloroplasts.

\section{Decay curves of luminescence evoked by white light}

The decay of luminescence, measured in the circulating-flow system, depends on the intensity of exciting light. At low intensities (up to about $20 \mathrm{ergs} / \mathrm{cm}^{2} \cdot \mathrm{sec}$ ) both luminescence "rise" and decay curves appear to be rectangular hyperbolae, indicating bimolecular kinetics. At higher light intensities the shape changes and "rise" and decay curves become relatively much steeper. The shape of the steep part cannot be determined by the flow method. In Fig. 3 decay curves of Chlorella lumines-

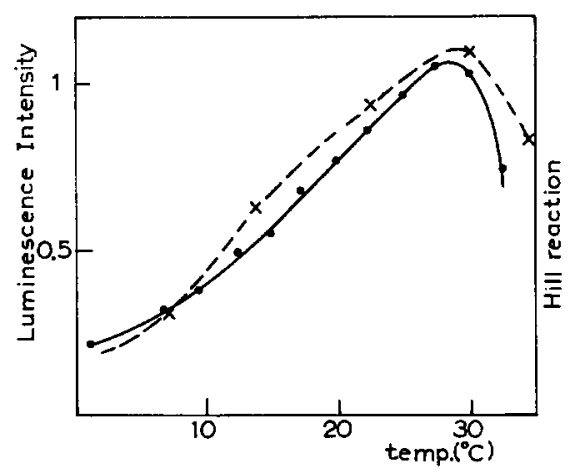

Fig. 2. Temperature curves of spinach chloroplast luminescence $(\cdot-\cdot)$ and Hill reaction capacity $(x--x)$, measured at approx. saturating light intensities.

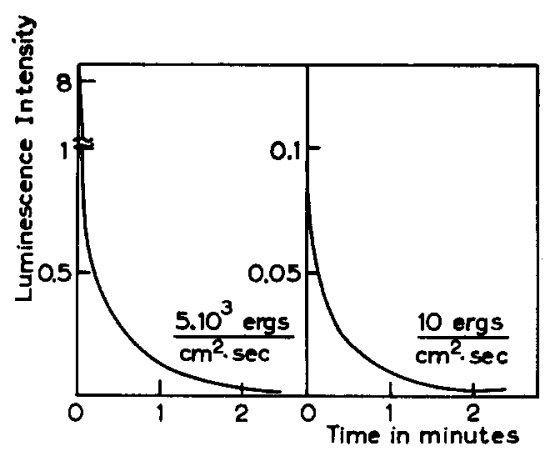

Fig. 3. Luminescence decay curves of Chlorella recorded after illumination with low and high light intensities.

cence are presented which were obtained after illumination with Io and $5 \cdot \mathrm{IO}^{3} \mathrm{ergs} /$ $\mathrm{cm}^{2} \cdot \mathrm{sec}$ incident light. Luminescence measured with the flow system thus seems to be composed of at least two different components, a fast and a slow one. Measurements with the phosphoroscope system showed that under our conditions of light intensity and temperature $\left(20^{\circ}\right)$ the "half life" of the shortest component was about o.I sec. Although the method permitted the measurement of luminescence with a half life as short as $5 \mathrm{msec}$, no such short-lived luminescence was detected. 


\section{Luminescence action spectra}

The experiments showed that, in some cases, monochromatic light produced luminescence values far greater than those obtainable with white light. To study this phenomenon luminescence action spectra were determined with Chlorella, Synechococcus and spinach chloroplasts.

In Fig. 4 luminescence action spectra of Chlorella and Synechococcus are presented using monochromatic light obtained from a grating monochromator. The intensity of exciting light was such that only the slow component was present. Luminescence at these light intensities was found to be a linear function of the exciting light intensity. It is clear from this figure that, as in the case of the efficiency of Chlorella photosynthesis ${ }^{17}$, the efficiency of Chlorella luminescence shows a steep decline on the long wavelength side of chlorophyll absorption.

For Synechococcus the maximum activity is not located at the chlorophyll $a$ maximum, but on the long-wavelength side of the phycocyanin absorption maximum. Action spectra for photosynthesis of blue-green algae ${ }^{15,18}$ are of a similar shape. The luminescence action spectrum thus has the same general shape as that obtained by ARNOLD AND ThOMPSON ${ }^{4}$.

In photosynthesis the activity resulting from absorption at wavelengths of low activity (approx. $700 \mathrm{~m} \mu$ with Chlorella and approx. $680 \mathrm{~m} \mu$ with Synechococcus) can be enhanced by simultaneous illumination with light of shorter wavelength ${ }^{17,19}$. A similar enhancement was not observed with luminescence at the weak intensities used for the determination of these action spectra. The activity produced by the two light beams together equalled the sum of the luminescence values obtained if the light beams were applied separately.

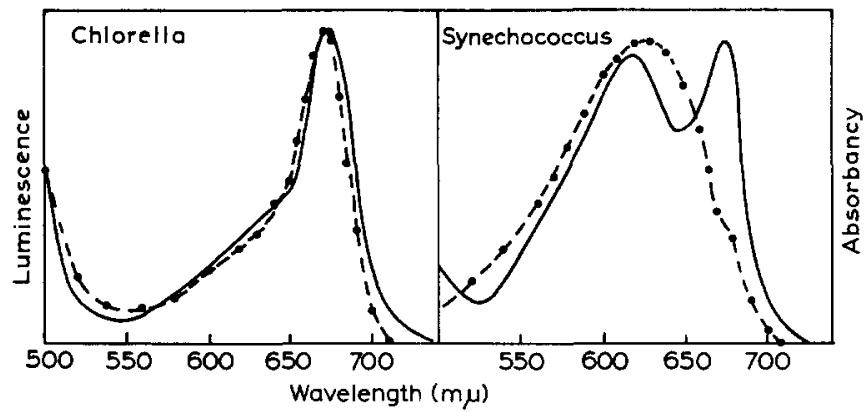

Fig. 4. Low intensity luminescence action spectra (-- - ) and absorption spectra (- -$)$ of Chlorella and Synechococcus showing inactivity of the major fraction of chlorophyll in Synechococcus, and a "long-wavelength drop" in Chlorella. Neither enhancement nor "quenching" was found at these light intensities $\left(<20 \mathrm{ergs} / \mathrm{cm}^{2} \cdot \mathrm{sec}\right)$.

\section{Suppression of luminescence by supplementary red light}

An attempt to detect an enhancement of luminescence at higher intensities of incident light led to the discovery of a decrease in luminescence if light absorbed by "long-wavelength chlorophyll" was added to that absorbed at shorter wavelengths. This luminescence decrease occurs only if the light intensities of both beams exceed a certain minimum value, usually of the order of $20-40 \mathrm{ergs} / \mathrm{cm}^{2} / \mathrm{sec}$ with Chlorella and of 50-I00 ergs $/ \mathrm{cm}^{2} / \mathrm{sec}$ with Synechococcus. If the intensities of both beams were reduced by interposing neutral filters, the effect disappeared. In Fig. 5 luminescence 
decrease is demonstrated with Chlorella if light beams of 659 and $700 \mathrm{~m} \mu$ are superimposed, while with Synechococcus it is shown that $680-\mathrm{m} \mu$ light decreases luminescence excited by $6 \mathrm{I}^{5} \mathrm{-m} \mu$ light. In neither case can a decrease be observed if $645-\mathrm{m} \mu$ light is added to the standard beam $\left(659\right.$ or $\left.6 \mathrm{I}_{5} \mathrm{~m} \mu\right)$. As the decrease in luminescence is equivalent to a luminescence quenching, it will be referred to as the "quenching effect".

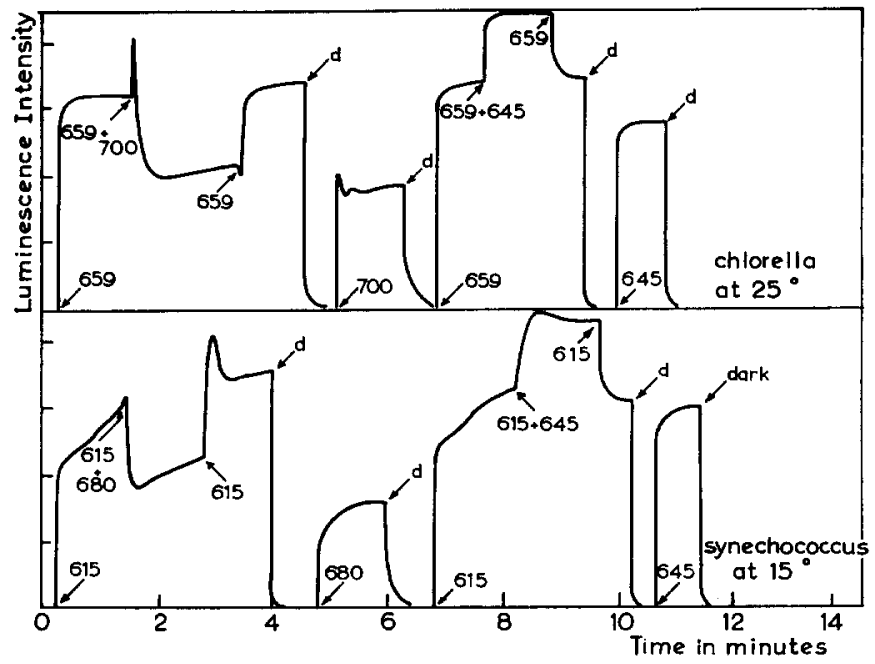

Fig. 5. Luminescence "quenching effect" in Chlorella and Synechococcus cells at intensities of incident light of approx. $200 \mathrm{ergs} / \mathrm{cm}^{2}$. sec. Addition of light absorbed by "long-wavelength chlorophyll $a$ " to light absorbed at shorter wavelengths results in a decrease in luminescence. Addition of other "promoting" light, however, results in an increase in luminescence. The numbers indicate the wavelength maxima of the interference filters. In Chlorella, the figure shows a sharp overshoot before "quenching" occurs.

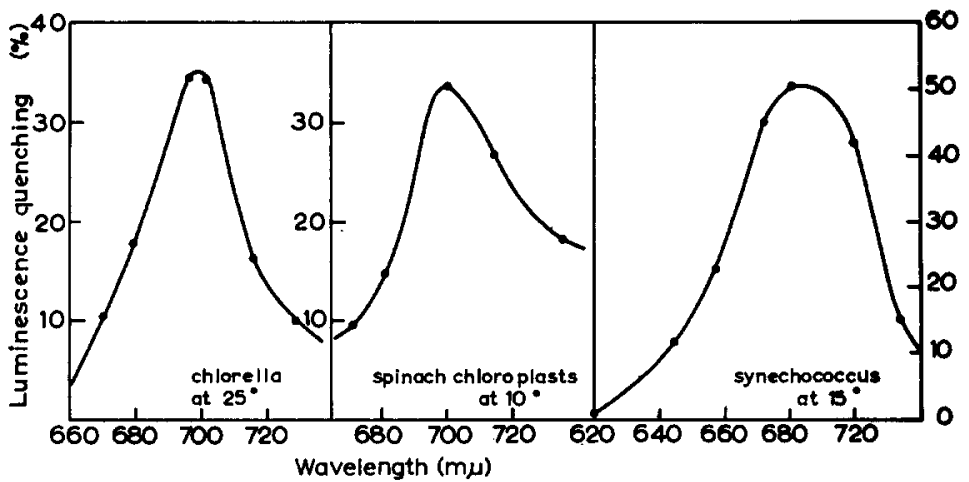

Fig. 6. Action spectra of the luminescence "quenching" effect in Chlorella, Synechococcus and spinach chloroplasts. Quenching is expressed as percentage decrease of measured luminescence

(value due to the sum of both light beams in the absence of a quenching effect $=$ I0o).

\section{Action spectrum of the "quenching effect"}

In Fig. 6 the action spectrum of the "quenching effect" is given for Chlorella, Synechococcus and spinach chloroplasts. With Chlorella and spinach chloroplasts a wavelength of $659 \mathrm{~m} \mu$, absorbed mainly by chlorophyll $b$ and "short-wavelength chlorophyll $a$ ", was chosen as reference light. With Synechococcus the wavelength 
was 6 I $5 \mathrm{~m} \mu$, absorbed mainly by phycocyanin. Fig. 7 demonstrates that with Chlorella and spinach chloroplasts the maximum quenching effect is around $700 \mathrm{~m} \mu$, while with Synechococcus it is around $680 \mathrm{~m} \mu$.

The "quenching effect" concerns mainly the fast luminescence component. An action spectrum of Synechococcus luminescence excitation, taken $7 \mathrm{sec}$ after illumination, shows the same general shape as the action spectrum of the "quenching effect", (Fig. 7).

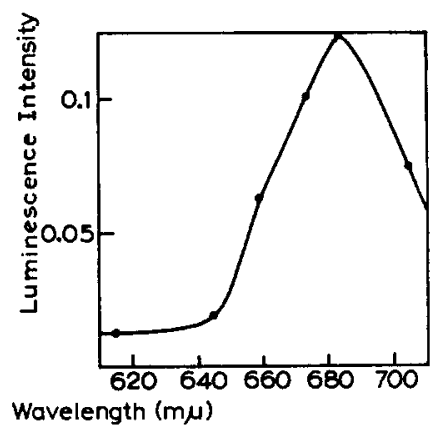

Fig. 7. Action spectrum of the slow component (determined 7 sec after the end of illumination) of Synechococcus luminescence, measured at illumination intensities above the critical quenching intensity.

At low temperatures the "quenching effect" is much more marked. With Chlorella at $4^{\circ}$ addition of $680-\mathrm{m} \mu$ light to $659-\mathrm{m} \mu$ light resulted in a marked decrease in luminescence, while at room temperature only a slight decrease occurred. With Synechococcus addition of light of a wavelength as short as $645 \mathrm{~m} \mu$ resulted in a "quenching" of luminescence excited by $6 \mathrm{I}_{5} \mathrm{~m} \mu$ at $4^{\circ}$, while addition of $590-\mathrm{m} \mu$ light resulted in the normal increase in luminescence.

Luminescence thus seems to be excited by absorption in one pigment system and can be suppressed by simultaneous absorption in another pigment system.

In the green alga Chlorella and in spinach chloroplasts the luminescence-promoting or "p" system can be assumed to consist of chlorophyll $b$ and the chlorophyll $a$ types $\mathrm{C}_{a} 673$ and $\mathrm{C}_{a} 680$, while the system responsible for luminescence "quenching" ("q" system) consists mainly of a chlorophyll $a$ type absorbing around $700 \mathrm{~m} \mu$ (tentatively called $\mathrm{C}_{\mathrm{a}}$ 695). In the blue-green alga Synechococcus the " $\mathrm{p}$ " system appears to consist of phycocyanin and a minor fraction of chlorophyll (defined here as $\mathrm{C}_{\mathrm{a}} 673$ and part of $\mathrm{C}_{\mathrm{a}} 68 \mathrm{o}$ ), while the " $\mathrm{q}$ " system consist of the major fraction of $\mathrm{C}_{\mathrm{a}} 680$ and $\mathrm{C}_{\mathrm{a}} 695$.

A study of the red absorption band of chlorophyll reveals that probably more than three types of this pigment occur in vivo ${ }^{23}$. The band width used in the luminescence experiments, however, is too large to permit an exact location of band maxima.

\section{Luminescence emission spectra}

ARNOLD AND DAvidson ${ }^{3}$ measured the emission spectrum of chlorophyll afterglow in vivo and found it to be similar to the emission spectrum of chlorophyll fluorescence. It is known from fluorescence studies ${ }^{20,21}$ that in Chlorella light of about $700 \mathrm{~m} \mu$ absorbed by chlorophyll is effective in emitting fluorescence of about $730 \mathrm{~m} \mu$. The yield of this fluorescence is low at room temperature but increases greatly upon cooling to the temperature of liquid nitrogen. With Synechococcus a separate $720-\mathrm{m} \mu$ 
fluorescence band can be detected even at room temperature. In view of these results, we investigated the possibility that long-wavelength chlorophyll absorption might also result in afterglow emission with a maximum around $730 \mathrm{~m} \mu$. In these experiments (at room temperature) with Chlorella interference filters (maximum transmission 680 and $725 \mathrm{~m} \mu$ ) were placed between luminescence detection vessel and multiplier. The ratio luminescence emitted at $680 \mathrm{~m} \mu$ to luminescence emitted at $725 \mathrm{~m} \mu$ was found to be the same, whether luminescence was excited by absorption mainly in the "p" system ( $659 \mathrm{~m} \mu)$ or by absorption mainly in the "q" system ( $703 \mathrm{~m} \mu$ interference filter plus Schott 2 mm RG8).

With Synechococcus, interposition of an RG8 filter (absorbing the 680-m $\mu$ luminescence peak and transmitting beyond $695 \mathrm{~m} \mu$ ) between multiplier and luminescence detection vessel decreased luminescence by the same fraction whether it was excited mainly in the "p" (6I5 $\mathrm{m} \mu)$ or mainly in the "q" system (700 $\mathrm{m} \mu)$. Thus, both with Chlorella and Synechococcus luminescence emitted in a wavelength region around $720 \mathrm{~m} \mu$ does not show a proportional increase as a result of predominant absorption in the " $\mathrm{q}$ " system. The results strongly suggest that only absorption in the " $\mathrm{p}$ " system results in luminescence. The activity around $700 \mathrm{~m} \mu$ is thus due to the "tail" of the "p" system.

\section{Luminescence of photosynthetic bacteria}

With the photosynthetic bacteria Rhodospirillum rubrum and Chromatium strain D, luminescence detected with the flow system was extremely weak. The intensity did not exceed $\mathrm{I} \%$ of the luminescence values of green plants measured under similar conditions of $\mathrm{CO}_{2}$ content, exciting light intensity and temperature, and based upon a comparable chlorophyll content. Flushing the cuvette with nitrogen did not result in a pronounced enhancement of luminescence.

Luminescence was excited by irradiation through interference filters with a maximum transmission at 800,860 and $880 \mathrm{~m} \mu$. Its intensity was approximately proportional to the absorption at these wavelengths. No measurable "quenching effect" was detected upon addition of supplementary light absorbed at the longwavelength maximum of bacteriochlorophyll (B89o) to light absorbed at shorter wavelengths (B80o or $\mathrm{B} 850$ ).

\section{DISCUSSION}

As the Hill reaction and luminescence compete for the excitation energy absorbed by the photosynthetic pigments, it may be expected that when the Hill reaction in chloroplast suspensions is initiated or accelerated by addition of oxidants, luminescence intensity decreases. As shown in a precedent section, such a decrease was indeed observed.

No competition is observed if the parameters temperature and intensity of actinic light are considered. Fig. 2 shows the same temperature dependency of luminescence and Hill reaction: both processes are irreversibly inhibited after prolonged heating to $65^{\circ}$. Also, it was found that the effect of storage at room temperature on the luminescence and Hill reaction capacity is similar. In Fig. Ib it can be seen that light saturation of chloroplast luminescence occurs in the same intensity region as light saturation of the Hill reaction. It thus seems plausible to assume that in 
both processes the same enzyme system is involved. We are thus led to suggest that chlorophyll afterglow in vivo is mainly a chemiluminescence, caused by recombination of products resulting from photolysis of water.

\section{Reversible oxidation of chlorophyll a}

Decomposition of water as a result of light absorbed by chlorophyll may be brought about by reversible oxidation of this pigment. In organic solution in vitro, a reversible oxidation of this sort can be obtained by consecutive addition of ferric ions and excess ferrous ions ${ }^{22}$. The absorption spectrum of the "oxy" form of chlorophyll $a$ in methanol ${ }^{24}$ is analogous to that of the photobleached form of chlorophyll in air-free methanol ${ }^{25}$. This may indicate that the reversible photobleaching represents a reversible photooxidation of chlorophyll. Also, the reversible photobleaching of chlorophyll by quinones at - $196^{\circ}$ may be seen as a photooxidation ${ }^{26}$.

Reduction of the chemically oxidised and photooxidised forms into chlorophyll results in emission of chemiluminescence. A similar relation between reversible oxidation and luminescence was observed with bacteriochlorophyll ${ }^{13}$.

The mechanism for decomposition of water in chloroplasts may thus be regarded as follows: Chlorophyll is excited by light absorption, the excited molecule losing an electron and becoming oxidised. The electron reduces a chemical compound $\mathrm{X}$, located close to the chlorophyll molecule. The oxidised chlorophyll is of a sufficiently high potential to decompose a closely adjacent water molecule. The electron of water finally reduces "oxy" chlorophyll. This electron might be regarded as being fed directly into the ground level of the chlorophyll molecule. In schematic form:

$$
\begin{aligned}
\mathrm{Chl} \stackrel{\mathrm{li}_{\mathrm{p}}}{\rightarrow} \mathrm{Chl}^{*} \rightarrow \mathrm{Chl}^{+}+\mathrm{e} \\
\mathrm{e}+\mathrm{X}_{\text {ox }} \rightarrow \mathrm{X}_{\mathrm{red}} \quad \mathrm{Chl}^{+}+\mathrm{H}_{\mathbf{2}} \mathrm{O} \rightarrow \mathrm{Chl}+\mathrm{H}^{+}+(\mathrm{OH})
\end{aligned}
$$

Oxygen evolution can result from $4(\mathrm{OH}) \rightarrow 2 \mathrm{H}_{2} \mathrm{O}+\mathrm{O}_{2}$.

In this scheme four $(\mathrm{OH})$ radicals (or related chemical products) are needed to produce one oxygen molecule.

Back reactions occur by a recombination of $(\mathrm{OH})$ left over in insufficient concentration to produce $\mathrm{O}_{2}, \mathrm{H}^{+}$and an electron from chlorophyll. Oxidised chlorophyll may acquire an electron from reduced $\mathrm{X}$. This electron might be regarded as fed into an excited state of chlorophyll: chemiluminescence occurs ${ }^{13}$ in the form of fluorescence from this excited state. We will not consider here whether the excited state concerns the first excited singlet or triplet state.

Whether such a mechanism (which may also explain the chemiluminescence of chlorophyll, Mg-phthalocyanine and metal porphyrins in heated peroxides ${ }^{11,12}$ ), can occur depends entirely on the separation in space or time of the electron leaving chlorophyll towards $\mathrm{X}$ and the electron entering chlorophyll from water. This separation, most probably, is brought about by a difference in shape between the electron distribution clouds (molecular orbitals) of the ground and of the excited states of the big flat chlorophyll molecule. Evidence for this contention may be seen in the following:

Reversible oxidation ${ }^{24}$ and chemiluminescence ${ }^{12}$ do not occur with the metal-free pheophytins, porphyrins or phthalocyanin. Reversible photochemical reduction of 
chlorophyll and bacteriochlorophyll ${ }^{27}$, however, proceeds almost as well, or even better, in the absence of the central magnesium atom as in the presence of it. This suggests that departure of an electron from the chlorophyll molecule is facilitated by the presence of the central magnesium atom, while entry of an electron is a property mainly affected by the shape of the conjugated ring structure.

Absorption of light energy by a chlorophyll molecule lifts an electron from the ground level to an excited level of the pigment. Absorption thus leaves an open space in the ground level during the time the electron lingers in some excited (singlet or triplet) level, before returning to the ground level with emission of light. If the space in the ground level is filled by an external electron (e.g. donated by ascorbic acid in the experiments in vitro ${ }^{27}$ ), reduced chlorophyll results. If no such external electron is available, but the electron from the excited level leaves the chlorophyll molecule via the central magnesium atom to reduce some solvent molecule, oxidised chlorophyll results.

In the molecular skeleton the most probable site at which the ground state can be filled with external electrons is determined by the shape of the molecular orbital of the ground state. A charge separation obtained in this way will not be effective in a random distribution of reactants as is present in solutions, but only in the specialised structure of the chloroplast, due to chloroplast proteins and other constituents. In this context it may be remarked that in etiolated leaves the capacity for photosynthesis was found to appear at approximately the same time as the formation of the "long-wavelength chlorophyll" and other structural changes 28

A continuous raising of electrons from a potential level of water decomposition to that of reduction of $\mathrm{X}$ is obtained if recombination is effectively prevented by a draining of electrons from $\mathrm{X}$ into the photosynthetic system.

\section{Intact photosynthesizing cells}

The experiments with Chlorella and Synechococcus showed that the observed afterglow results from excitation in the " $p$ " system only, while simultaneous irradiation with light absorbed by the "q" system results in a decrease in luminescence intensity. This can be explained by the assumption that only simultaneous absorption by the " $p$ " and " $q$ " systems results in an effective use of absorbed light energy for photosynthesis. Duysens et al..$^{29}$ attributed, in the red alga Porphyridium, oxidation of a cytochrome to a pigment system which is probably similar to our "q" system, while reduction of this cytochrome occurred as a result of irradiation with light of the same wavelengths as those exciting our " $p$ " system (cf. also HiLl AND BeNDaLL ${ }^{30}$ and WITT et al. ${ }^{31}$ ). In view of these results we suggest that our "quenching effect" is based on cytochrome oxidation, while the "promotion" is related to cytochrome reduction.

It was suggested above that the splitting of water resulted in reduction of a chemical compound $\mathrm{X}$. If the redox value of $\mathrm{X}$ exceeds that of the cytochrome, the latter will be reduced by $X$. Thus, in living cells, absorption of light by the " $\mathrm{p}$ " system results in reduction of $\mathrm{X}$ and subsequently in reduction of cytochrome. If no additional light, absorbed by the "q" system, is applied, recombination of the electron from reduced $\mathrm{X}$ with $(\mathrm{OH})$ and $\mathrm{H}^{+}$results in luminescence (the fast luminescence component). The lower redox potential of cytochrome as compared with $\mathrm{X}$ does not preclude some molecules of $\mathrm{X}$ being reduced by cytochrome as a back reaction. 
Reduced $\mathrm{X}$ molecules are then again available for recombination and light emission, resulting in the slow luminescence component.

The results in the paragraph on Decay curves of luminescence evoked by white light may be explained as follows: At low actinic light intensities, the stationary concentration of reduced $\mathrm{X}$ is very low, since all the $\mathrm{X}^{-}$is used to reduce cytochrome. Hence only a slow luminescence component results. At higher actinic light intensities (absorbed mainly by the " $\mathrm{p}$ " system) all the X and cytochrome will be in the reduced form. As a consequence, the occurrence of both a fast and a slow component is to be expected.

The "p" system thus supplies reducing power. Wesseis ${ }^{32}$ showed that the Hill reaction proceeds with various quinones of redox potentials ranging from -0.44 to $-0.05 \mathrm{~V}$, but not higher. Photochemical reduction of dyes occurred with dyes of a redox potential up to $-0.03 \mathrm{~V}$. System "p" thus exerts reducing capacity from the redox potential of water decomposition to approximately - $-0.05 \mathrm{~V}$. Compound $\mathrm{X}$, closest to the " $\mathrm{p}$ " chlorophyll $a$ pigment, should have a redox potential of the order of - o.I V. No conclusions can be drawn about the chemical nature of this compound from luminescence experiments. So far plastoquinone seems to be the most likely component to act as $\mathrm{X}^{33}$.

Light absorbed by the " $q$ " system is supposed to oxidise cytochrome, originally reduced by " $p$ " absorption. The electron of cytochrome is then removed from the " $\mathrm{p}$ " system. At high light intensities, when both $\mathrm{X}$ and cytochrome are in the reduced state after illumination with " $\mathrm{p}$ " light, oxidation of cytochrome by additional " $\mathrm{q}$ " absorption results in a decrease in the stationary concentration of reduced $\mathrm{X}$ and hence in a decrease mainly of the fast component of luminescence (Fig. 6). The slow component survives (Fig. 7).

Except at the long-wavelength side of the red chlorophyll absorption band, absorption of " $p$ " and "q" systems overlaps at all wavelengths.

If a single beam of white light is used as actinic light, the increase in luminescence as a function of light intensity due to "p" absorption is compensated (or even overcompensated) by the decrease in luminescence due to " $q$ " absorption. This results in a light curve as given in Fig. Ia. When a copper sulfate filter is placed in the white incident beam, removing light of a wavelength longer than $680 \mathrm{~m} \mu$, luminescence increases by about $50 \%$.

The following remarks may be made on the function of the " $q$ " system in photosynthesis. FRENCH AND FORK ${ }^{34}$ found a photochemical system to be active in producing respiratory stimulation. MCLEOD ${ }^{35}$ observed a requirement for inorganic phosphate for the enhancement effect. The action spectra of these phenomena closely resemble that of our " $q$ " system. This is also true of the action spectrum of photophosphorylation catalysed by phenazinemethosulfate $e^{36}$ and reduction of pyridine nucleotides ${ }^{29}$. One may conclude, therefore, that absorption by the "q" system offers reducing power sufficient to reduce phosphopyridine nucleotide (redox potential about $0.3 \mathrm{~V}$ ), for formation of high energy phosphates and for stimulation of respiration.

Photosynthetic reduction capacity thus seems to be spread over the two pigment systems: system "p" produces reducing power from below the redox potential of splitting of water (about $-0.8 \mathrm{~V}$ ) to $-0.05 \mathrm{~V}$, resulting in vivo in reduction of $\mathrm{X}$, and hence in reduction of a cytochrome (presumably cytochrome $f$, redox potential about - $0.4 \mathrm{~V})$; system "q" produces reducing power from the level of oxidation of 
cytochrome to the level of reduction of phosphopyridine nucleotide. The electron from cytochrome thus reduces phosphopyridine nucleotide.

Combined absorption of " $p$ " and " $q$ " systems results in a raising of an electron from water to a level sufficient to produce reduced phosphopyridine nucleotide. From this level, possibly with the help of high energy phosphates also formed by "q" absorption, the energy flows into the dark processes of photosynthesis, resulting in reduction of $\mathrm{CO}_{2}$ to carbohydrate. Fig. 8 shows a highly schematic picture in which the energy levels in the electron pathway are given.

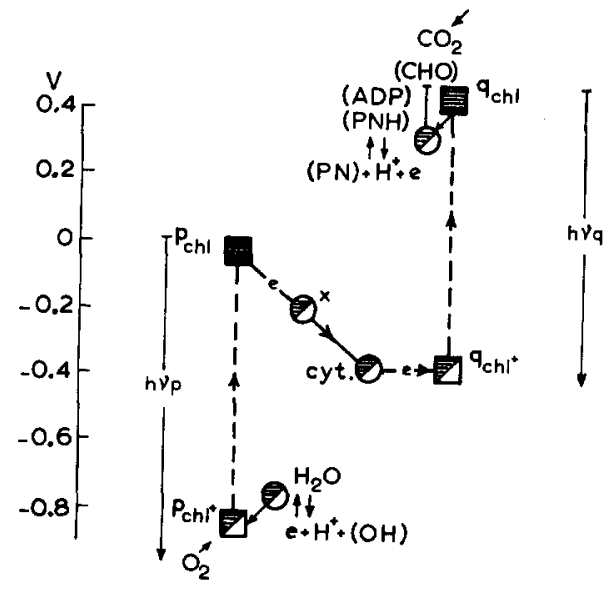

Fig. 8. Energy scheme of light reactions in photosynthesis. Due to absorption in the "p" chlorophyll $a$ system, the electron of water is raised to a sufficiently high potential level to reduce $\mathrm{X}$ and, ultimately, cytochrome. Luminescence occurs as back reaction from $\mathrm{X}$ and recombination of water. Draining of electrons from $X$ by addition of Hill reactants (chloroplasts) or by oxidation of cytochrome (absorption by the "q" chlorophyll a system) prevents recombination and decreases luminescence. Absorption by " $\mathrm{q}$ " chlorophyll $a$ lifts the electron to the reduced phosphopyridine nucleotide level and ultimately, with the help of high energy phosphate, to the carbohydrate level.

There are the following indications that the action of the " $q$ " system also proceeds via reversible oxidation of chlorophyll $a$. The luminescence "quenching effect" is much more marked at $4^{\circ}$, i.e. when the photosynthetic and Hill reaction rates are low and luminescence of chloroplasts due to the " $p$ " system is weak. Hence this effect, assumed to be based on cytochrome oxidation, is less sensitive to temperature than the other processes. This conclusion is in agreement with the results of CHANCE AND NisHIMURA $^{37}$ who observed absorption changes at - $196^{\circ}$ upon illumination of the photosynthetic bacterium Chromatium, ascribed by them to cytochrome oxidation. MULLER AND WITT ${ }^{38}$ observed similar changes at $-I_{50^{\circ}}$ with green algae. Thus cytochrome oxidation seems to occur without a measurable activation energy.

Experiments with Anacystus by KOK AND HoCH ${ }^{36}$ showed that reversible changes of a chlorophyll type absorbing around $700 \mathrm{~m} \mu$ could be produced by light absorption as well as by addition of ferricyanide. A redox potential of $-0.46 \mathrm{~V}$ was measured for this chlorophyll type, which seems to be similar to that exciting our "q" system.

Experiments of GOEDHEER ${ }^{39}$ demonstrated that in purple bacteria the bacteriochlorophyll spectra in the near infrared undergo, upon addition of ferri-ferrocyanide mixtures of $-0.5 \mathrm{~V}$, changes which closely resemble a difference spectrum as measured

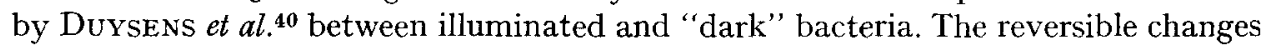
of the $890-\mathrm{m} \mu$ band are similar to the changes obtained with reversible oxidation of bacteriochlorophyll in methanol. The experiments of KoK and GoEDHEER suggest that a reversible oxidation of chlorophyll $\mathrm{C}_{\mathbf{a}} 695$ and bacteriochlorophyll B 890 occurs as a result of illumination. A cytochrome, present in the close neighbourhood of these 
pigments, becomes oxidised upon illumination. Accordingly, we may write the electron exchange mechanism occurring in the chlorophyll-containing organisms:

$$
\begin{aligned}
& \mathrm{C}_{\mathrm{a}} 695 \stackrel{\mathrm{h} \boldsymbol{v}_{\mathrm{q}}}{\longrightarrow} \mathrm{C}_{\mathrm{a}} 695^{\star} \rightarrow \mathrm{C}_{\mathrm{8}} 695^{+}+\mathrm{e} \\
& \mathrm{e}+(\mathrm{PN})+\mathrm{H}^{+} \rightarrow(\mathrm{PNH}) \quad \mathrm{C}_{\mathrm{a}} 695^{+}+\text {cytochrome }_{\mathrm{red}} \rightarrow \mathrm{C}_{\mathrm{a}} 695+\text { cytochrome }_{\mathrm{ox}}
\end{aligned}
$$

The reversible bleaching of chlorophyll and bacteriochlorophyll becomes apparent only if all the cytochrome is present in the oxidised state, e.g. in suitable conditions of the bacteria (Duysens) or under the influence of high-intensity light flashes (KoK).

With respect to chlorophyll $a$ type $\mathrm{C}_{\mathrm{a}} 695$, BRoDy ${ }^{20}$ showed that the properties of this chlorophyll resemble those of a chlorophyll aggregate, probably a dimer, in organic solution. It seems also possible, however, that the particular properties of $\mathrm{C}_{\mathrm{a}} 695$ in vivo (low fluorescence yield, red shift and change in shape of absorption band) are due to chlorophyll-cytochrome interaction instead of chlorophyll-chlorophyll interaction.

In chloroplast suspensions absorption by the " $q$ " system at room temperature soon fails to suppress luminescence emitted by the "p" system, as it does in intact cells. This indicates an uncoupling of the " $p$ " and " $q$ " systems in chloroplasts, which might be due to either a diffusion or oxidation of cofactors needed for reduction of $\mathrm{CO}_{2}$, or to structural changes in the chloroplast. With respect to the latter, the " $\mathrm{p}$ " and " $q$ " systems must be separated topographically to such an extent, that no energy transfer by inductive resonance occurs between them. Such a transfer would cause the "q" system to be an energy sink for all absorbed light energy and thus prevent photosynthesis. Structural changes, such as those caused by swelling, might well disrupt the link between the two systems.

According to our scheme, in the Hill reaction "proper", i.e. photochemical decomposition of water and production of reduced compounds with a redox potential not exceeding $0.05 \mathrm{~V}$, only light absorbed in the " $\mathrm{p}$ " system is involved. LosADA, WHATLEY AND ARNON ${ }^{41}$ also suggested that only one of two pigment systems was involved in the liberation of oxygen from water. In accordance with our findings, BECKER, SHEFNER AND GROSS ${ }^{42}$ found that the capacity for the Hill reaction of 2,6-dichlorophenolindophenol could be maintained for several hours at room temperature.

\section{Photosynthetic bacteria}

Whether or not, in photosynthetic bacteria, two pigment systems occur, similar to the ones in green plants, cannot be decided as yet. The following indications do, however, suggest the possibility of existence of two pigment systems: The near infrared bacteriochlorophyll difference spectrum, obtained upon addition of ferricyanide, was found to be composed of two components: a reversible bleaching of part of B 890 and a reversible shift of part of B 800 (see ref. 39). The former component might be ascribed to a pigment system similar to the "q" system of green plants. In Rhodospirillum rubrum this system covers more than $90 \%$ of the total amount of bacteriochlorophyll molecules. Absorption of light by intact bacteria results in cytochrome oxidation ${ }^{\mathbf{4 3}}$ and reduction of pyridine nucleotide ${ }^{\mathbf{4}}$. In bacterial chromatophore suspensions photophosphorylation and reduction of pyridine nucleotide can be observed ${ }^{45}$. No Hill reaction, similar to that of green plants (reduction 
of quinones and dyes) has been found to occur in these suspensions. Hence, a possible " $p$ " system in photosynthetic bacteria should function differently from that in green plants. If the reversible shift of B 800 represents the equivalent of a "p" system, absorption by this system most probably does not result in decomposition of water. Luminescence of photosynthetic bacteria at room temperatures is very weak. This phenomenon, and the observation that it is excited also by absorption in B 890 , suggests that luminescence is emitted by the equivalent "q" system.

\section{REFERENCES}

1 B. L. Strehler and W. Arnold, J. Gen. Physiol., 34 (1951) 809.

2 W. Arnold, in F. H. Johnson, The Luminescence of Biological Systems, Publ. of A.A.A.S., I95.5, p. 47 .

3 W. Arnold and J. B. Davidson, J. Gen. Physiol., 37 (1954) 677.

4 W. Arnold and J. Thompson, J. Gen. Physiol., 39 (I956) 3 I I.

5 W. E. Arthur and B. L. Strehler, Arch. Biochem. Biophys., 70 (1957) 507.

B B. L. Strehler and V. Lynch, Arch. Biochem. Biophys., 7o (1957) 527.

7 W. Arnold and H. Sherwood, J. Phys. Chem., 63 (I959) 2.

${ }^{8}$ G. Tollin and M. Calvin, Proc. Natl. Acad. Sci. U.S., 43 (1957) 87.

$9 \mathrm{~J}$. E. Brugger and J. Franck, Arch. Biochem. Biophys., 75 (I958) 456.

10 H. O. Albrecht, W. C. Denison, L. G. Livingston and C. E. Mandeville, J. Frankl. Inst., 268 (1959) 278.

$11 \mathrm{~J}$. Rothemund, J. Am. Chem. Soc., 60 (1938) 2005.

12 H. Linschitz, in W. MCElroy and B. Glass, A symposium on Light and Life, Johns Hopkins Press, Baltimore, I96I, p. I73.

13 J. C. Goedheer and G. R. Vegt, Nature, I93 (I962) 875.

14 P. B. Sweetser, C. W. Todd and R. T. Hersh, Biochim. Biophys. Acta, 5 I (196I) 509.

15 F. Haxo and L. R. Blinks, J. Gen. Physiol., 33 (I950) 389.

16 A. S. Holt and C. S. French, Arch. Biochem., I9 (I948) 368.

17 R. Emerson and E. I. Rabinowitch, Plant Physiol, 35 (I960) 477.

${ }^{18}$ L. M. N. Duysens, Thesis, Utrecht, I952.

19 G. C. MCLeod and C. S. French, Carn?gie Inst. Wash. Yearbook, 59 (1960) 336.

20 S. S. Brody, Science, 128 (1958) 838.

21 W. L. Butler, Avch. Biochem. Biophys., 93 (I96I) 4 I 3.

22 E. I. Rabinowitch and J. Weiss, Proc. Roy. Soc. (London) A, 162 (1937) 251.

23 J. B. Thomas, Biochim. Biophys. Acta, 59 (I962) 202.

24 J. C. GoEDHEER, Biochim. Biophys. Acta, 27 (1958) 478; unpublished experiments.

25 R. Livingston and V. A. Ryan, J. Am. Chem. Soc, 75 (I953) 2176.

26 H. Linschitz and J. Rennert, Nature, I69 (I952) I93.

27 A. A. KRasnowskil and G. P. Brin, Doklady Acad. Nauk U.S.S.R., 89 (1953) 527.

28 J. C. Goedheer, Biochim. Biophys. Acta, 53 (I96I) 420.

29 L. M. N. Duysens, J. Amesz and B. N. KAmp, Nature, Igo (I96I) 5 Io.

30 R. Hill and F. Bendall, Nature, I86 (1960) I36.

31 H. T. Witt, A. Mullfr and B. Rumberg, Nature, 192 (I961) 967.

32 J. S. C. Wessel.s, Philips Research Repts., 9 (1954) I 40.

38 E. R. Redfearn and J. Friend, Natuve, IgI (I96I) 806.

34 C. S. French and D. C. Fork, Carnegie Inst. Wash. Yearbook, 60 (Ig6I) $35 \mathrm{I}$.

35 G. C. McLeod, Science, 133 (I96r) 192.

${ }^{36}$ B. KoK and G. Hoch, in W. D. McElroy and B. Glass, A Symposium on Light and Life, Johns Hopkins Press, Baltimore, I96r, p. 397.

37 B. Chance and M. Nishimura, Proc. Natl. Acad. Sci. U.S., 46 (1960) I9.

38 A. Muller and H. T. WITT, Nature, I 89 (I96I) 944.

39 J. C. Goedheer, Biochim. Biophys. Acta, $3^{8}$ (1960) $3^{89}$.

40 L. M. N. Duysens, W. J. Huiskamp, J. J. Vos and J. M. van der Hart, Biochim. Biophys. Acta, I9 (I956) I 88 .

41 M. Losada, F. R. Whatlet and D. J. Arnon, Natuve, igo (Ig6i) 606.

42 M. J. Becker, A. M. Shefner and J. A. Gross, Nature, I93 (Ig62) 92.

${ }^{43}$ L. M. N. Duysens, Carnegie Inst. Wash. Yearbook, 53, (1953-1954) I66.

44 J. M. Olson, Brookhaven Symposia in Biology, 1957, p. 3 I6.

45 A. W. Frenkel, J.Am. Chem. Soc., 80 (I958) 246. 